\title{
Reduction of four-and-a-half LIM-protein 2 expression occurs in human left ventricular failure and leads to altered localization and reduced activity of metabolic enzymes
}

Esta Bovill, MRCS, ${ }^{a}$ Steven Westaby, FRCS, PhD, ${ }^{\mathrm{b}}$ Alastair Crisp, MA, ${ }^{\mathrm{c}}$ Shiney Jacobs, $\mathrm{PhD},{ }^{\mathrm{a}}$ and Tony Shaw, $\mathrm{PhD}{ }^{\mathrm{a}}$

\begin{abstract}
Objective: We sought to identify changes in four-and-a-half LIM-protein 2 levels and location in human cardiomyocytes during the transition from compensated aortic stenosis to left ventricular failure. We also sought to characterize four-and-a-half LIM-protein 2 binding with the metabolic enzymes phosphofructokinase 2, adenylate kinase, and creatine kinase $\mathrm{M}$ isoform during this transition and their consequential subcellular localization in failing human ventricles.
\end{abstract}

\begin{abstract}
Methods: Left ventricular biopsy specimens from selected patients undergoing aortic valve replacement for aortic stenosis were allocated to one of 2 groups: (1) nondilated with preserved left ventricular function (nonfailing group, $\mathrm{n}=16$ ) and (2) grossly dilated with poor left ventricular function (failing group, $\mathrm{n}=15$ ). These were compared with a control group of unused donor hearts $(n=6)$. Protein levels and subcellular localization were determined by means of Western blotting and immunofluorescence. Four-and-a-half LIM-protein 2 binding to adenylate kinase, creatine kinase $\mathrm{M}$ isoform, or phosphofructokinase 2 was studied by means of coimmunoprecipitation. Phosphofructokinase 2, adenylate kinase, and creatine kinase $\mathrm{M}$ isoform activities were assayed in protein extractions.
\end{abstract}

Results: Four-and-a-half LIM-protein 2 levels were preserved in nonfailing hypertrophied hearts but reduced by $53 \%$ in failing hearts. The pattern of four-and-a-half LIM-protein 2 staining was disrupted in failing hearts: fourand-a-half LIM-protein 2 was lost from the sarcomere but present in the perinuclear Golgi apparatus complex. Phosphofructokinase 2, adenylate kinase, and creatine kinase M isoform coimmunoprecipitated in vitro and colocalized with four-and-a-half LIM-protein 2 in both hypertrophied and failing hearts. Phosphofructokinase 2 and adenylate kinase activities were reduced to $77 \%$ and $58 \%$ of normal values in compensated aortic stenosis, with phosphofructokinase 2 activity decreased further to $56 \%$ of normal value in failing hearts, but creatine kinase activity remained unchanged.

Conclusions: Altered four-and-a-half LIM-protein 2 expression in heart failure is associated with disruption of the normal subcellular localization of phosphofructokinase 2, adenylate kinase, and creatine kinase $\mathrm{M}$ isoform and reduced activity of phosphofructokinase 2 and adenylate kinase, which might have important consequences for myocardial energy metabolism in heart failure.

Clinical decision making regarding the optimal timing for aortic valve replacement is an inexact science. At the time of clinical presentation, patients with aortic stenosis already manifest a broad spectrum of myocardial functional change. Left ventricular (LV) morphology ranges from asymptomatic compensatory hypertrophy to cavity dilation with symptoms of advanced heart failure. During valve replacement

\footnotetext{
From the Department of Medicine, ${ }^{\mathrm{a}}$ University College London, London, United

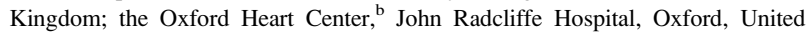
Kingdom; and the Department of Physiology, Anatomy and Genetics, ${ }^{\mathrm{c}}$ University of Oxford, Oxford, United Kingdom.

Drs E. Bovill, S. Jacobs, and T. Shaw are supported by grants from the British Heart Foundation.

Received for publication April 14, 2008; revisions received Aug 13, 2008; accepted for publication Sept 1, 2008.

Address for reprints: Esta Bovill, MRCS, c/o Oxford Heart Centre, John Radcliffe Hospital Headley Way, Headington, Oxford OX3 9DU, United Kingdom (E-mail: ebovill@doctors.net.uk).

J Thorac Cardiovasc Surg 2009;137:853-61

0022-5223/\$36.00

Copyright (C) 2009 by The American Association for Thoracic Surgery

doi:10.1016/j.jtcvs.2008.09.006
}

surgery, impaired LV function can negatively influence survival, ${ }^{1}$ particularly when myocardial energetics might have already deteriorated before the period of ischemic arrest. Access to human myocardium during surgical intervention provides a unique opportunity to correlate myocyte molecular changes to the LV phenotype, which can be characterized beforehand by means of detailed cardiac imaging.

Abnormal recruitment of metabolic enzymes to the cardiac sarcomere, via known interactions between sarcomeric proteins and stretch-dependent signaling molecules may play an important role in the deterioration of myocardial function in heart failure. ${ }^{2,3}$ Four-and-a-half LIM-protein 2 (FHL2) is thought to act as a specific adaptor protein coupling metabolic enzymes to sites of high energy consumption in the sarcomere through interaction with titin/connectin. ${ }^{4}$ Titin forms an elastic scaffold spanning more than $1 \mu \mathrm{m}$ from a Z-disc to the M-band. ${ }^{4}$ Localization of metabolic enzymes to metabolically active regions within the sarcomere is necessary for energy provision during contraction. ${ }^{5}$ Mutations in $F H L 2$ have already been identified as predisposing to 


$$
\begin{aligned}
& \text { Abbreviations and Acronyms } \\
& \begin{aligned}
\text { ADP } & =\text { adenosine diphosphate } \\
\text { AK } & =\text { adenylate kinase } \\
\text { ATP } & =\text { adenosine triphosphate } \\
\text { ATPase } & =\text { adenosine triphosphatase } \\
\text { CK-M } & =\text { creatine kinase isoform M } \\
\text { DAPI } & =4^{\prime} \text {-6-Diamidino-2-phenylindole } \\
& \text { dihydrochloride } \\
\text { ERK } & =\text { extracellular signal-regulated kinase } \\
\text { FHL2 } & =\text { four-and-a-half LIM-protein } 2 \\
\text { LV } & =\text { left ventricular } \\
\text { PBS } & =\text { phosphate-buffered saline } \\
\text { PFK2 } & =\text { phosphofructokinase } 2 \\
\text { SDS } & =\text { sodium dodecylsulfate }
\end{aligned}
\end{aligned}
$$

idiopathic dilated cardiomyopathy. ${ }^{2}$ Studies in cardiac sarcomeres have shown FHL2 localized to broad striations at the Z-disk. ${ }^{4}$ This distinctly localized FHL2 couples adenylate kinase (AK), creatine kinase isoform $\mathrm{M}(\mathrm{CK}-\mathrm{M})$, and phosphofructokinase 2 (PFK2) to titin. ${ }^{4}$

Cardiac contraction requires efficient energetics. Communication defects between adenosine triphosphate (ATP)producing and ATP-consuming sites are a major contributor to deficient energetics in heart failure. Reduced activity of $\mathrm{CK}, \mathrm{AK}$, and glycolytic enzymes, which collectively facilitate ATP delivery and promote removal of adenosine diphosphate (ADP), $\mathrm{Pi}$, and $\mathrm{H}^{+}$from cellular adenosine triphosphatases (ATPases), has been described in the failing heart. ${ }^{6}$ Energy transfer through AK, CK, and glycolytic enzymes is an adaptive mechanism, but the compensatory potential of these systems is reduced in the failing heart. ${ }^{6}$

In the failing heart the overall ATP turnover rate is similar to the normal value, but there are changes in the kinetics of ATP use, suggesting that a smaller ATP pool is involved. ${ }^{6}$ This could result from an altered localization or deficient phosphotransfer of adenine nucleotides. ${ }^{7}$ Decreased energetic efficiency has been observed in CK-knockout hearts, ${ }^{8}$ which supports a critical role for phosphotransfer reactions in myocardial energetics. ${ }^{6}$

PFK2 is a bifunctional enzyme that controls the synthesis

\begin{tabular}{|c|c|c|c|c|c|}
\hline & Donor & Nonfailing & Failing & $\begin{array}{c}P \\
\text { value* }\end{array}$ & $\begin{array}{c}\text { Normal } \\
\text { range }\end{array}$ \\
\hline No. of patients & 6 & 16 & 15 & NA & \\
\hline Male/female sex & $4 / 2$ & $9 / 7$ & $7 / 8$ & & \\
\hline Diagnosis & NA & AS & AS & & \\
\hline Age (y) & $36 \pm 10$ & $62 \pm 15$ & $60 \pm 18$ & NS & \\
\hline $\begin{array}{l}\text { LV end-diastolic } \\
\text { diameter }(\mathrm{cm})\end{array}$ & ND & $4.8 \pm 0.5$ & $6.4 \pm 0.5$ & .002 & $3.5-5.6$ \\
\hline $\begin{array}{l}\text { LV end-systolic } \\
\text { diameter }(\mathrm{cm})\end{array}$ & ND & $2.8 \pm 0.4$ & $4.3 \pm 0.5$ & .004 & $2.5-4.1$ \\
\hline $\operatorname{LVEF}(\%)$ & ND & $58 \pm 5$ & $35 \pm 5$ & .009 & $55-75$ \\
\hline $\begin{array}{l}\text { Peak transvalvular } \\
\text { gradient }(\mathrm{mm} \mathrm{Hg})\end{array}$ & ND & $83 \pm 25$ & $86 \pm 26$ & .83 & \\
\hline NYHA class & NA & 1.4 & 2.9 & .011 & \\
\hline
\end{tabular}
and degradation of fructose 2,6-bisphosphate. Fructose 2,6bisphosphate is a potent stimulator of 6-phosphofructo-1kinase, a key glycolytic enzyme. Cardiac PFK2 is activated by adrenaline, insulin, or high workload. This correlates with increased glycolysis under the same conditions. ${ }^{9}$ PFK2 levels or activity have not been measured in heart failure, but reduced PFK2 activity could reduce glycolytic flux, resulting in impaired cardiac function. Because FHL2 is known to couple PFK2, AK, and CK-M to titin at metabolically active regions within the sarcomere, we sought to further investigate this adaptor role in human myocardium
TABLE 1. Preoperative echocardiographic and NYHA assessment of patient groups

taken from healthy hearts, together with hearts from patients with aortic stenosis but normal contractility, and myocardium taken from patients with aortic stenosis, ventricular dilation, and established heart failure.

\section{MATERIALS AND METHODS Patient Selection}

Under a protocol approved by the Oxford Medical Research Ethics Committee, myocardial biopsy specimens were obtained during aortic valve replacement for aortic stenosis in 2 well-defined age- and sex-matched categories of consenting patients. The first group was characterized as having aortic stenosis with echocardiographically and clinically (New York Heart Association) well-preserved LV function. Primarily given diagnoses during unrelated surgical work-up, indications for surgical intervention in this group primarily comprised optimization before anesthetic, tight stenosis, or both. The second group had aortic stenosis with impaired (decompensated) LV function, together with established clinical heart failure (Table 1). Patients with coronary artery disease were excluded. Medical regimens were noted, with no overall difference between the surgical groups. All patients underwent preoperative transthoracic echocardiographic analysis to quantify peak transvalvular gradient, LV function, and LV dimensions. LV dimensions were measured on parasternal M-mode views taken at the level of the chordae tendinae. The heart failure group contained 15 patients. Those with preserved LV function were designated the nonfailing group $(n=16)$. A control group of LV biopsy specimens was obtained from structurally normal unmatched donor hearts that were not used for transplantation $(\mathrm{n}=6)$.

The biopsy specimens, weighing at least $2 \mathrm{~g}$, were taken from the interventricular septum in the LV outflow tract beneath the right coronary cusp and away from conduction tissue. All myocardial biopsy specimens were immediately snap-frozen in liquid nitrogen and stored at $-80^{\circ} \mathrm{C}$.

\section{Protein Extraction and Western Blotting}

Protein was extracted into buffer $(8 \mathrm{~mol} / \mathrm{L}$ urea, $2 \%$ sodium dodecylsulfate [SDS], $10 \mathrm{mmol} / \mathrm{L} \mathrm{CHAPS}$, and $250 \mathrm{mmol} / \mathrm{L}$ Tris, $\mathrm{pH} 8.0$ ), and the protein concentration of each sample was determined by using the Bio-Rad (Hercules, Calif) protein assay. FHL2 and enzyme levels in myocardial 
protein isolates were detected by means of Western blotting. Aliquots (100 $\mu \mathrm{g}$ ) of protein were separated by means of electrophoresis on $12 \%$ SDSpolyacrylamide gels and blotted onto nitrocellulose according to standard protocols. FHL2 was detected with a mouse mAb, and standard mouse antibodies against PFK2, AK, and CK-M (AbCam, Cambridge, Mass) were used to detect these enzymes. Gel loading was confirmed by reprobing blots with a $\beta$-actin antibody (AbCam). Blots were probed with a horseradish peroxidase-conjugated secondary antibody (DakoCytomation, Glostrup, Denmark) and analyzed by means of chemiluminescence (Amersham Biosciences, Piscataway, NJ) on a densitometer (Bio-Rad) to allow calculation of the intensity of each target band relative to $\beta$-actin by using proprietary software.

\section{Immunofluorescence}

Cryosections $(5 \mu \mathrm{m})$ were cut from frozen tissue and fixed in $4 \%$ paraformaldehyde before solubilization in $0.1 \%$ triton X-100 in phosphate-buffered saline (PBS). Sections were blocked with $10 \%$ fetal calf serum in PBS and $0.1 \%$ Tween 20 . Standard staining procedures were used. All washes were performed with PBS and $0.1 \%$ Tween 20 . Slides were counterstained with 4'-6-diamidino-2-phenylindole, dihydrochloride (DAPI); AlexaFluor488-Phalloidin (Molecular Probes, Eugene, Ore); or both. A rabbit anti-FHL2 polyclonal antibody ${ }^{10}$ was detected with a goat anti-rabbit IgG conjugated to AlexaFluor546 (Molecular Probes). The Golgi apparatus complex was stained with anti-Golgin p97 (Molecular probes). Standard protocols were used for immunofluorescent detection of AK, PFK2, and CK-M. Control staining with only secondary antibodies was performed on each section. Confocal microscopy was performed with a Bio-Rad confocal microscope. A stack of $1.0-\mu \mathrm{m}$-thick sections were recorded, and at least 3 different fields were examined for each sample.

\section{Coimmunoprecipitation Assay}

Protein was extracted into IP buffer (10 mmol/L N-2-hydroxyethylpiperazine-N-2-ethanesulfonic acid [pH 7.4], $100 \mathrm{mmol} / \mathrm{L} \mathrm{KCl}, 5 \mathrm{mmol} / \mathrm{L}$ $\mathrm{MgSO}_{4}, 0.5 \% \mathrm{NP}-40,50 \mu \mathrm{mol} / \mathrm{L} \mathrm{ZnSO}_{4}$, and $1 \mathrm{mmol} / \mathrm{L}$ dithiothreitol) and separated by means of centrifugation at $13,000 \mathrm{~g}$ for 5 minutes at $4^{\circ} \mathrm{C}$. Protein concentration in the supernatant was measured as above. For each assay, $750 \mu \mathrm{g}$ of protein was incubated with $2.0 \mu \mathrm{g}$ of the appropriate antibody for 2 hours at $4{ }^{\circ} \mathrm{C}$, after which $10 \mu \mathrm{L}$ of $50 \%$ Protein A agarose was added and the mixture was incubated overnight at $4{ }^{\circ} \mathrm{C}$ with rotation. Beads were washed 3 times in IP buffer and 3 times in IP buffer with $250 \mathrm{mmol} / \mathrm{L}$ $\mathrm{KCl}$, and then protein was resuspended in Laemmli buffer, denatured, and electrophoresed on a $12 \%$ SDS-polyacrylamide gel. Immunoblotting was performed as described above.

\section{Enzyme Assays}

Enzyme activities were measured from tissue extracted with PBS (pH 7.4), $0.5 \%$ NP- 40 , and $10 \%$ glycerol. All extracts were desalted with a 7000 molecular weight (MW) cutoff matrix in a 0.5 -mL spin column (Pierce, Inc, Cheshire, United Kingdom) to remove contaminating small molecules, such as sugars, nicotinamide adenine dinucleotide, reduced nicotinamide adenine dinucleotide, ADP, or ATP. Spectrophotometric enzyme-linked assay for PFK2 activity was performed as previously described. ${ }^{11} \mathrm{~A}$ luminescence-based $\mathrm{AK}$ assay kit (BioVision, Mountain View, Calif) was used for AK activity, according to the manufacturers' protocol; the initial AK assay was repeated in the presence of $50 \mu \mathrm{mol} / \mathrm{L}$ P1,P5-bis(adenosine)-5' -pentaphosphate, and activity measured in the presence of this inhibitor was subtracted from total activity to correct for any non-AK-mediated activity. CK activity was measured according to the protocol of Bishop and colleagues ${ }^{12}$ but with the addition of $50 \mu \mathrm{mol} / \mathrm{L}$ P1,P5-bis(adenosine)-5' -pentaphosphate A to inhibit AK activity.

\section{Statistical Analysis}

Data are expressed as means \pm standard deviation. Comparisons were performed by using the Student's $t$ test or analysis of variance with the post hoc Student-Newman-Keuls test where appropriate.

\section{RESULTS}

Changes in FHL2, AK, CK-M, and PFK2 Protein Levels in Cardiac Hypertrophy and Heart Failure

Western blot analysis (Figure 1, A) shows that FHL2 levels are maintained in hypertrophied LV tissue but reduced by $53 \%$ in failing hearts (Figure 1,B). Similarly, AK and PFK2 levels are decreased in failing hearts compared with those seen in the control group $(48 \%$ and $57 \%$ reduction, respectively; Figure 1, $C$ and $D$ ). There are no significant differences in CK-M levels between groups, although there is a trend toward reduced expression in failing hearts (Figure 1,E).

\section{Pattern of FHL2 Expression Is Disrupted in the Failing Ventricle}

The distinctive FHL2 cross-striation pattern is observed in normal and hypertrophied LV sections (Figure 2, $A$ and $B$ ), but this pattern is disrupted in failing LV sections: FHL2 is not localized to the sarcomere with a cross-striated appearance, but FHL2-specific staining is predominantly in cytoplasmic foci, many of which are adjacent to DAPI-stained nuclei and reminiscent of Golgi apparatus (Figure 2, C). Colocalization of FHL2 with Golgi apparatus is observed in the failing group (Figure 3). This was consistently found at each $1-\mu \mathrm{m}$ level in each section; in every failing LV section, the FHL2 signal was predominantly colocalized with the Golgi apparatus, although some of the Golgi apparatus had no FHL2 staining. This is in contrast to the FHL2 staining of the nonfailing groups, in which FHL2 was clearly seen only in the previously observed striated pattern. The negative controls (secondary antibody only) showed no distinct staining, and no Golgi apparatus localization of FHL2 was observed in the nonfailing group (data not shown).

\section{AK, CK-M, and PFK2 Interactions with FHL2}

FHL2 binds with AK, CK-M, and PFK in the normal left ventricle, and these interactions are maintained in hypertrophied and failing hearts despite the altered subcellular distribution of FHL2 (Figure 4). Immunofluorescence for AK, CK-M, and PFK2 (Figure 5) shows that all 3 enzymes mirror FHL2 localization to the Golgi apparatus in the failing heart; this pattern is observed at each level in all sections.

\section{Enzyme Activities}

PFK2 activity is reduced to $76 \%$ of control value in the hypertrophied left ventricle and further decreased to $56 \%$ in failing hearts. AK activity is decreased to $58 \%$ of control value in hypertrophied and failing hearts. CK-M activity is not altered in hypertrophied or failing hearts (Table 2).

\section{DISCUSSION}

FHL2 is a member of the four-and-a-half LIM domain protein subfamily found in the earliest identifiable cardiac precursor cells but restricted primarily to cardiomyocytes in the adult, ${ }^{13}$ where it is located mainly at the Z-disc. 

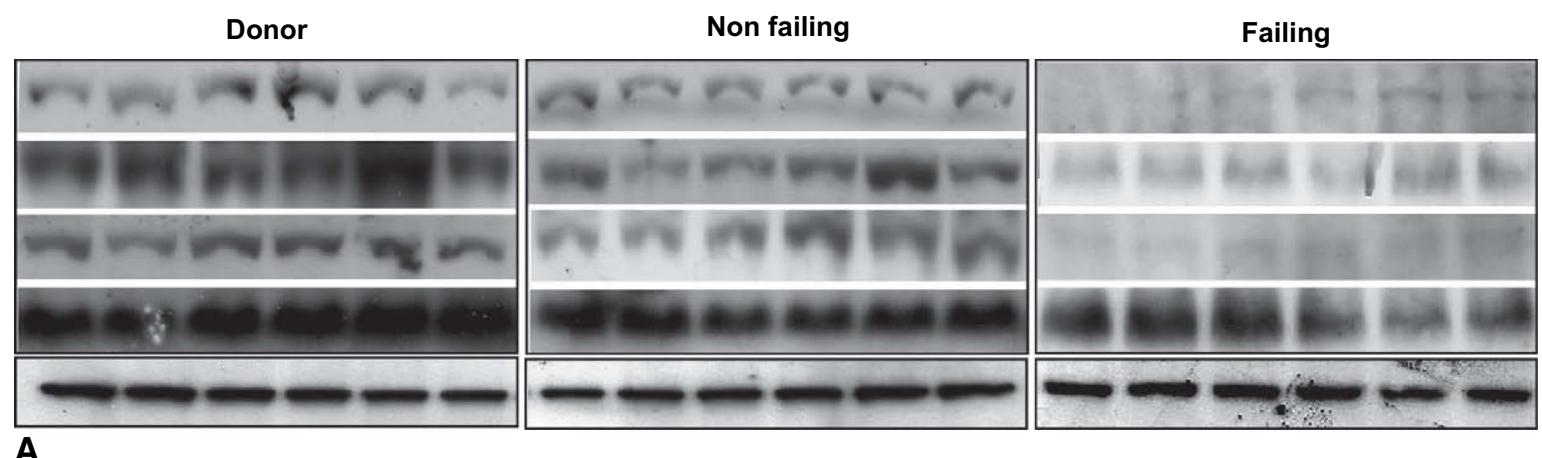

FHL-2

AK

PFK2

CK-M

$\beta$-Actin

FHL -2 / $\beta$ Actin

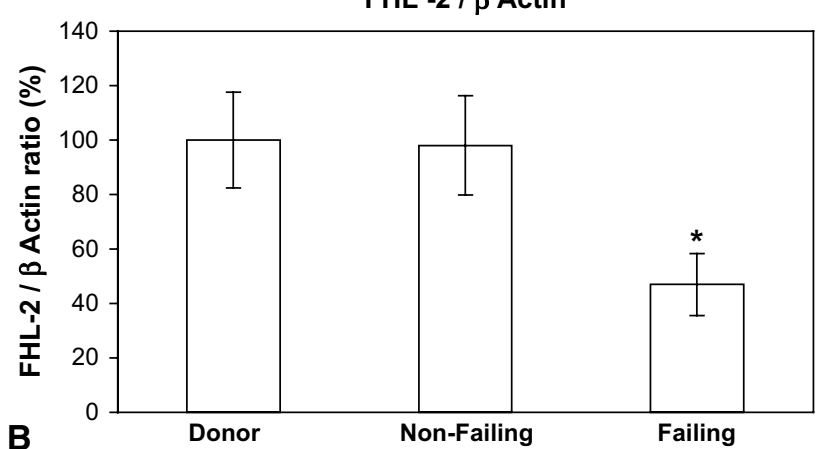

PFK2 / $\beta$ Actin

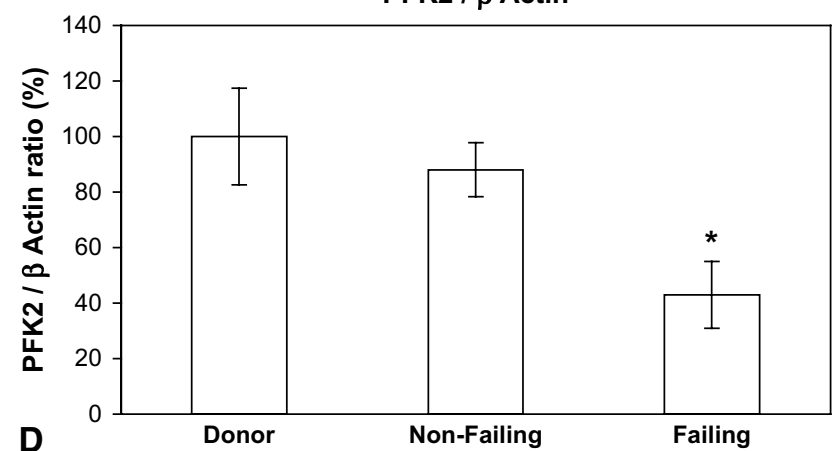

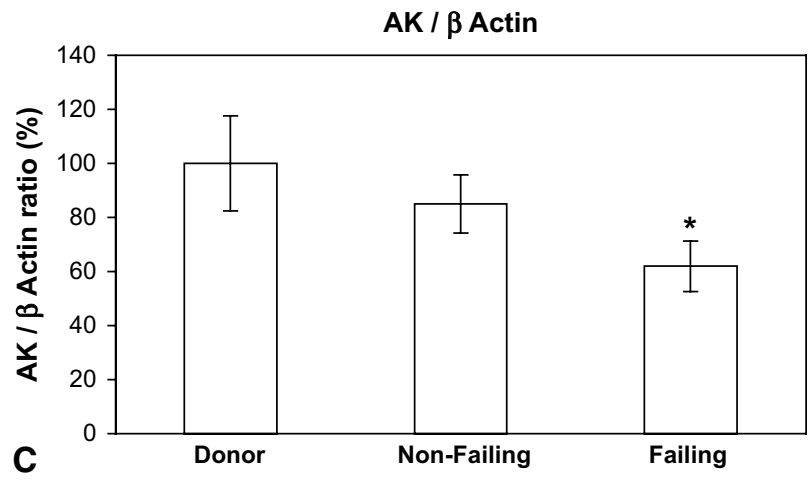

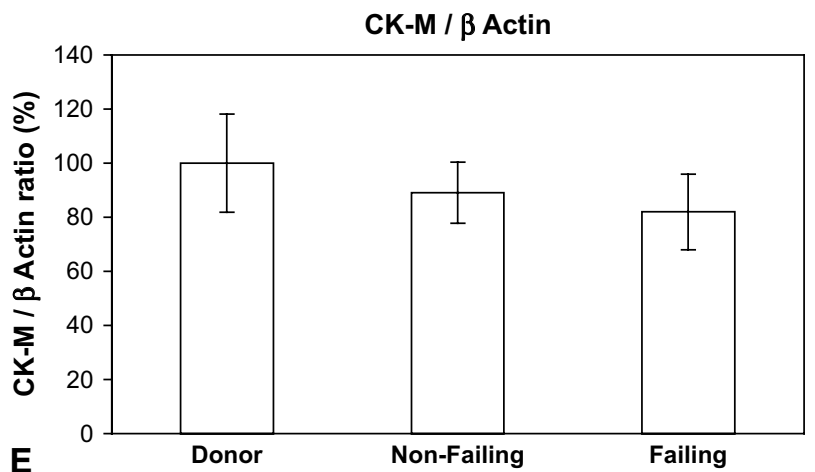

FIGURE 1. Four-and-a-half LIM-protein $2(F H L-2)$, adenylate kinase $(A K)$, and phosphofructokinase 2 (PFK2) levels are significantly decreased in the failing human heart. A, Representative Western blot of FHL2 in patients from the donor, nonfailing, and failing groups. $\beta$-Actin was used as a loading control. Each lane represents a different heart. A selection of patients is shown. Densitometric analyses of blots were performed, and the ratio of protein band intensity to $\beta$-actin band intensity was used to compare relative amounts of each protein. FHL2 levels were decreased by $53 \%$ compared with normal levels (B). AK levels decreased by $48 \%$ (C) and PFK2 levels decreased by $57 \%$ (E) compared with levels in the control group. CK-M levels decreased slightly (D), but this was not statistically significant. ${ }^{*} P<.05$.

Several LIM domain proteins have roles in the development and maintenance of cellular architecture through myofibril adherence to the cytoskeleton. The Z-disc localization of FHL2 is shared with muscle LIM protein, the expression of which is vital for the structural organization of myofibrils, with muscle LIM protein-deficient mice displaying disrupted cytoarchitecture, dilated cardiomyopathy, and heart failure. ${ }^{14}$ A mouse knockout for the LIM protein Alp has right ventricular cardiomyopathy. ${ }^{15}$

Lange and associates ${ }^{4}$ proposed that FHL2 acts as a specific adaptor protein to couple metabolic enzymes to sites of high energy consumption within the sarcomere through the interaction with titin. FHL2 mutations affect the binding to both N2B and its 2 regions of titin, leading to impaired recruitment of metabolic enzymes to the cardiac sarcomere and hence to cardiac failure. These observations led to the suggestion that cardiac dysfunction in heart failure might partly result from the interaction between sarcomere and stretch-dependent signaling molecules. ${ }^{2}$

The N2B region of titin not only determines the elastic properties of the sarcomere, it also relates to the ischemic stress and cardiac hypertrophy responses through its binding 


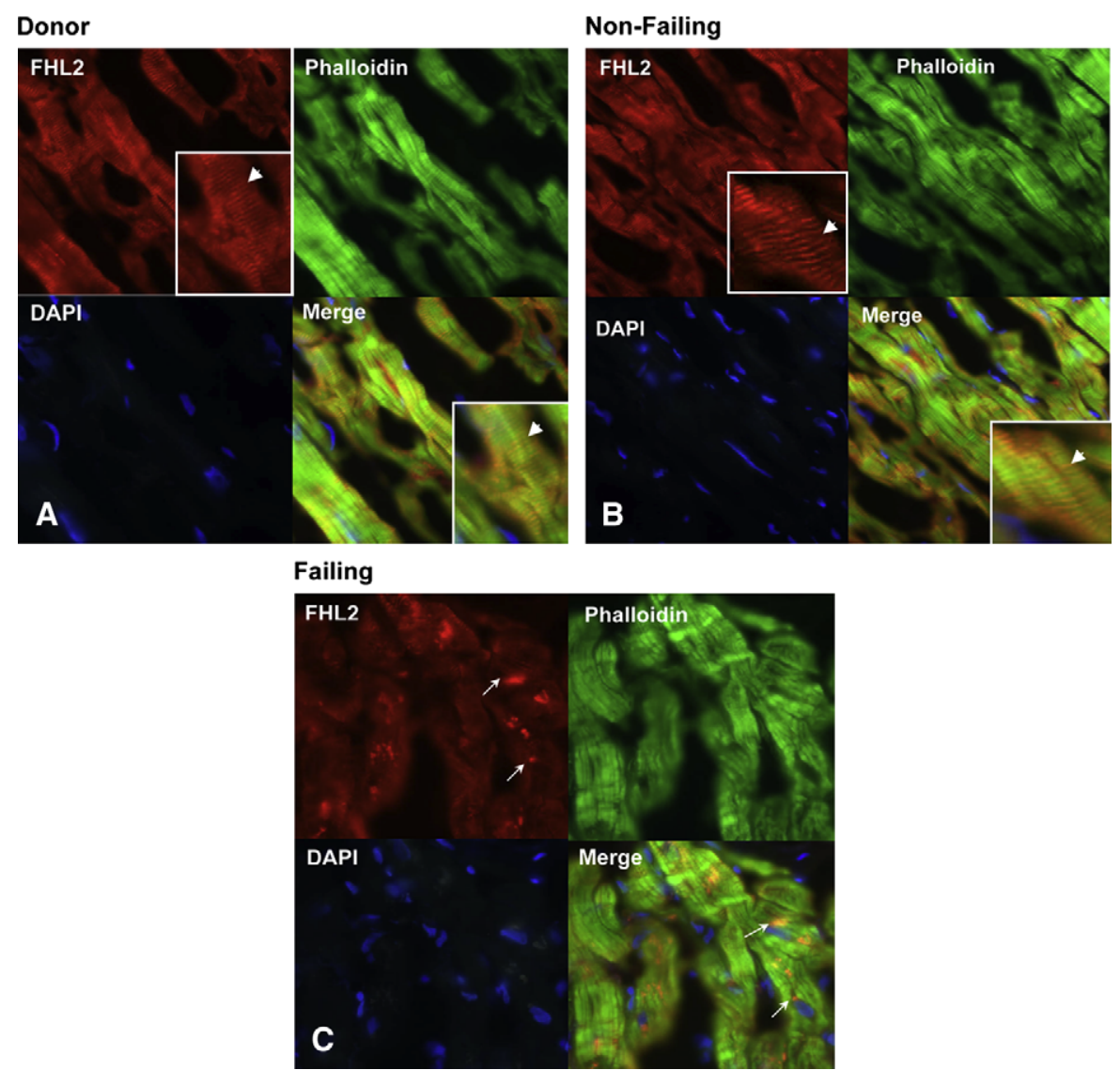

FIGURE 2. Four-and-a-half LIM-protein 2 (FHL2) accumulates in discrete cytoplasmic foci in failing human hearts. Representative immunofluorescence microscopic analysis of FHL2 in donor (A), nonfailing (B), and failing (C) left ventricular myocardium. FHL2 (red) in cardiomyocytes from patients with preserved myocardial function (nonfailing group) shows a regular staining pattern indicated by arrowheads and the enlarged section of the merge panel. FHL2 in cardiomyocytes from patients in the failing group is sequestered in discrete cytoplasmic foci (arrows). Phalloidin staining (green) was used to identify cardiomyocytes, and all sections were counterstained with DAPI (blue).

proteins $\alpha$ B-cristalin and FHL2, respectively. Radke and coworkers ${ }^{16}$ found that FHL2 protein was downregulated on deletion of its binding site within the N2B region of titin. These authors suggest that the interaction of N2B and FHL2 provides a novel regulatory pathway to control the balance of hypertrophy and atrophy in the heart. This suggestion is reinforced by the finding that patients with a mutation in the titin N2B region (S3799Y) have hypertrophic cardiomyopathy associated with an increased binding of FHL2 to titin. ${ }^{3}$ Titin therefore appears to be a key component in the assembly, force transmission, and maintenance of resting tension in the sarcomere and tethers many proteins, including FHL2, in the sarcomere. Accordingly, abnormalities in the sarcomeric proteins interacting with titin/connectin cause hereditary dilated cardiomyopathy.

Previous research into the role of FHL2 in the failing myocyte is limited. FHL2-null mice have greater cardiac hypertrophic responses compared with those of wild-type control animals after catecholamine infusion ${ }^{17}$ but not when subjected to pressure overload by means of aortic banding. ${ }^{13}$
FHL2 specifically attenuates the transcriptional activator function of extracellular signal-regulated kinase (ERK) because it blocks the mitogen-activated protein kinase kinase 1-ERK1/2-mediated hypertrophic response of the atrial natriuretic factor promoter. ${ }^{18}$ The disparate hypertrophic response in gene-targeted mice might reflect differences in signaling pathways involved in pressure overload and catecholamine-induced hypertrophy. However, FHL2 acts as both a transcriptional coactivator ${ }^{10}$ and corepressor. ${ }^{19}$ This might also be an explanation for different hypertrophic responses in FHL2 gene-targeted animals.

Localization of metabolic enzymes to metabolically active regions of the sarcomere is necessary for energy provision during contraction. ${ }^{5}$ This is demonstrated by a study from Wojtas and associates ${ }^{20}$ in which transgenic Drosophila constructed with glycolytic enzymes unable to localize within the sarcomeres of their flight muscles were unable to fly despite normal protein and activity levels. Diffusional ATP/ADP exchange does not allow rapid and efficient intracellular communication. ${ }^{5,6,21}$ Reactions catalyzed by 


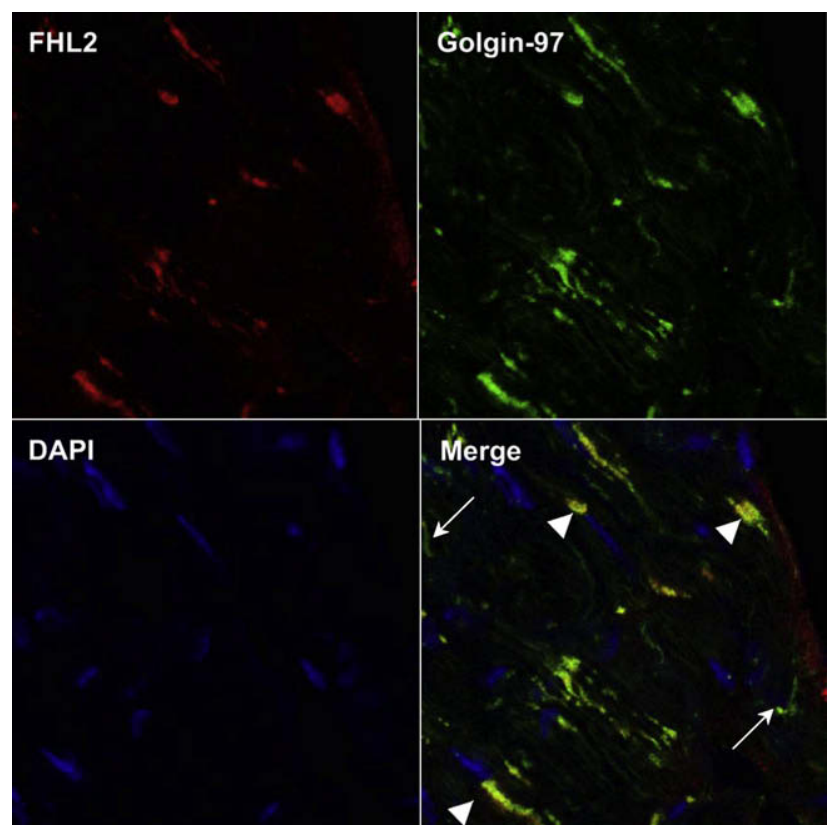

FIGURE 3. Four-and-a-half LIM-protein 2 (FHL2) colocalizes with the Golgi-specific marker Golgin 97 in cardiomyocytes from the failing group. Sections were stained for FHL2 (red) and Golgin97 (green) and counterstained with DAPI. The majority of FHL2 staining is present in the Golgi (arrowheads), but not all Golgi have FHL2 staining (arrows). A $1.0-\mu \mathrm{m}$ confocal section is shown.

creatine kinase, AK, and glycolytic enzymes facilitate transfer of high-energy phosphoryls to ATPases to promote communication between ATP-consuming and ATP-generating processes. ${ }^{6,21}$ Our hypothesis was that FHL2 plays an important role as an adaptor protein, coupling metabolic enzymes to titin, and that this situation might be disturbed in the transition between compensatory myocardial hypertrophy and heart failure. Although our histologic observations do not prove that FHL2 localization influences high energy metabolism or causes heart failure, the altered localization, along with the maintenance of FHL2 interactions with CK, $\mathrm{AK}$, and PFK2, suggests that FHL2 is worthy of further investigation, at least as a mediator of high energy metabolism in the septal wall.

Through its role as an adaptor protein, FHL2 might play a part in human cardiomyocyte contractility and is altered in heart failure. In clinically well-categorized patient populations with aortic stenosis, we showed significantly decreased FHL2 expression in failing hearts compared with that seen in those with well-preserved left ventricular function or in healthy control hearts (Figure 1). FHL2 joins an array of proteins with altered expression in heart failure; further work to clarify the level at which downregulation occurs will help determine the role of FHL2 in heart failure signaling. Nevertheless, our main hypothesis focused on the cytoarchitectural consequences of altered FHL2 expression. Consistent with

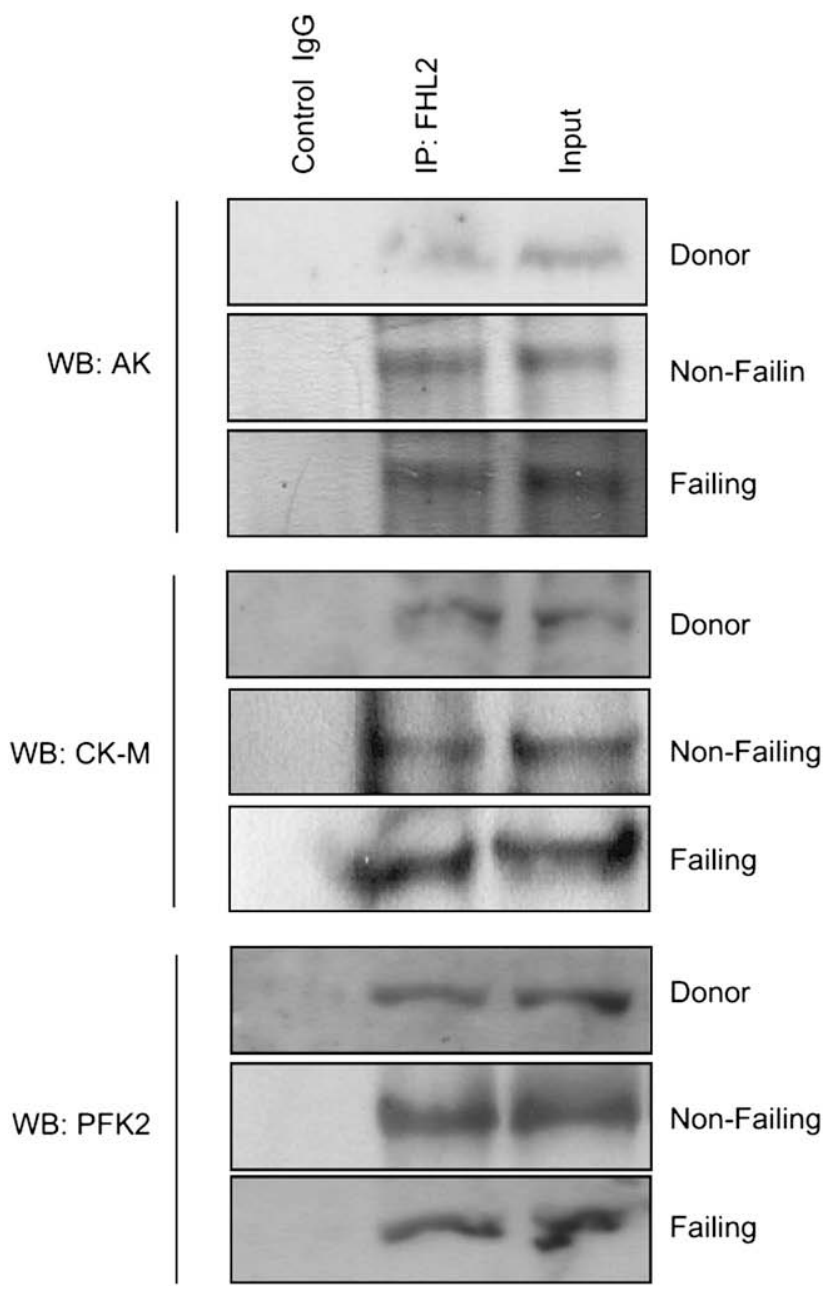

FIGURE 4. Adenylate kinase $(A K)$, creatine kinase isoform $\mathrm{M}(C K-M)$, and phosphofructokinase 2 ( $P F K 2)$ maintain their interaction with fourand-a-half LIM-protein 2 (FHL2) in the failing heart. Coimmunoprecipitation from extracts of patient hearts from each group shows that AK, CK-M, and PFK2 are in a complex with FHL2. A representative result is shown. Coimmunoprecipitations were performed from every sample. $W B$, Western blot.

previous studies, our findings confirmed the localization pattern of FHL2 for healthy hearts. We also showed, using coimmunoprecipitation from human tissue, that AK, CK-M, and PFK2 are bound to FHL2 at the sarcomere. Lange and colleagues ${ }^{4}$ have previously suggested a role for FHL2 as an adaptor molecule linking these enzymes to metabolically active compartments during contraction. Similar but spatially distinct roles for FHL2 as an adaptor/docking protein have been identified, including cell adhesion ${ }^{22}$ and linkage of voltage-gated ion channels to the cytoskeleton. ${ }^{23}$

Our results and those of Wojtas and associates ${ }^{20}$ indicate that the mechanisms that supply ATP to ATPases for muscle contraction require a highly organized cellular system. By comparing the subcellular localization of FHL2 in healthy 

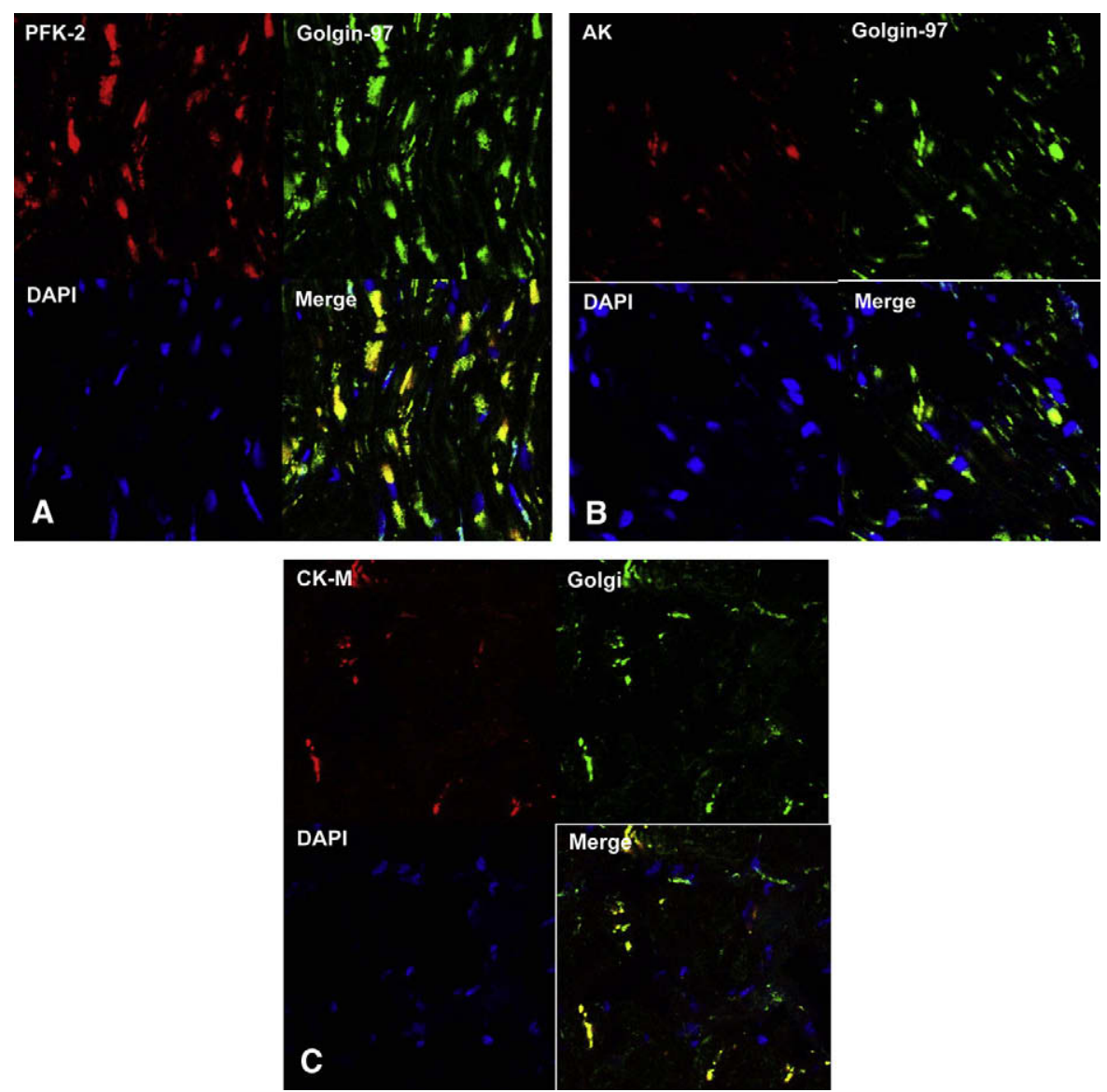

FIGURE 5. Adenylate kinase $(A K)$, creatine kinase isoform $\mathrm{M}(C K-M)$, and phosphofructokinase $2(P F K 2)$ are also present in the Golgi apparatus in the failing heart. PFK2, AK, and CK-M (A, B, and C, respectively) all colocalize with Golgin97 (green) in the Golgi apparatus of failing cardiomyocytes. Confocal sections of $1.0 \mu \mathrm{m}$ are shown. A representative result is shown. Staining was performed on every sample.

human hearts with that of hearts with aortic stenosis, we have shown that the orderly pattern in normal cardiomyocytes is significantly disrupted in the failing group (Figure 2) but not before the onset of failure in hypertrophied hearts. In failing cardiomyocytes FHL2 showed absent or severely diminished localization to the Z-lines, instead accumulating in the Golgi apparatus. AK, CK-M, and PFK2 remain bound to FHL2 in all groups. The significance of the coimmunoprecipitation result is that it suggests FHL2 sequesters these enzymes away from the sarcomere by means of direct interaction with them, as opposed to merely occupying the same subcellular location, as indicated by the immunofluorescence. In nonischemic heart failure N2B and N2BA cardiac titin isoforms had an N2BA/N2B expression ratio that was significantly increased despite unchanged ${ }^{24}$ or decreased ${ }^{25}$ total titin content. Such alterations of the N2B isoform, which binds FHL2, ${ }^{4}$ could contribute to the disruption of FHL2, AK, CK-M, and PFK2 localization we observed in failing cardiomyocytes.

The significance of Golgi apparatus accumulation is uncertain, but it might simply reflect altered intracellular protein transport in failing myocytes. Defective intracellular protein transport in heart failure can be seen by the inhibition of Rab1-mediated endoplasmic reticulum-to-Golgi transport in cardiomyocytes. ${ }^{26} \mathrm{~A}$ combination of altered titin isoform expression and defective intracellular transport might lead to the sequestration of FHL2, AK, CK-M, and PFK2 complexes in the Golgi apparatus as heart failure progresses.

The failing myocardium is characterized by changes in energy and substrate metabolism, although numerous human studies have failed to consistently elucidate the direction of alterations in glucose use. Several studies investigated the role of individual enzymes in pathologic hearts. We have

TABLE 2. AK, CK, and PFK2 enzyme activities expressed per milligram of extracted soluble protein

\begin{tabular}{lccc}
\hline Enzyme & Donor & Nonfailing (AS) & Failing (AS) \\
\hline AK & $5.72 \pm 1.03$ & $3.36 \pm 1.18^{*}$ & $3.34 \pm 1.27^{*}$ \\
PFK2 & $12.17 \pm 3.07$ & $9.34 \pm 2.87^{*}$ & $6.87 \pm 1.93^{*} \dagger$ \\
CK & $10.62 \pm 2.98$ & $10.77 \pm 3.01$ & $9.37 \pm 2.97$ \\
\hline
\end{tabular}

Enzyme activities are expressed as micromoles per minute per milligram. Values are presented as means \pm standard deviation. ${ }^{*} P<.05$ versus control groups. $\dagger P<.05$ versus the nonfailing group. 
shown reduced glycolytic enzyme activities overall, but AK activity has been shown to both increase and decrease ${ }^{6}$ in the septal wall of failing myocardium. These disparities might reflect a spectrum of responses to the degree of failure. Enhanced glycolysis as a source of ATP has been proposed to represent an adaptive mechanism in early heart failure; it is possible that this adaptation is undermined by mislocalization of glycolytic enzymes as decompensated heart failure progresses. Association of enzymes with other proteins might also influence their activities; the association of glycolytic enzymes and band-3 protein or tubulin ${ }^{27}$ reduces enzyme activity, whereas binding of 6-phosphofructo1-kinase to F-actin accelerates glycolytic flux in muscle. ${ }^{28}$ AK and PFK2 enzyme activities in our human samples were decreased, whereas CK activity tended to decrease, albeit insignificantly, despite preserved binding to FHL2. This implies allosteric activation of these enzymes is unusually, albeit indirectly, related to localization by a modulating protein other than FHL2. The influence of titin-enzyme interaction represents an important question in this context.

Furthermore, our results allow the distinction of 2 aspects of enzyme function in cardiac muscle: localization and catalytic activity. Catalytically active enzyme alone might not be sufficient for muscle contraction, rather enzymes must be properly localized to enable efficient function. Precisely how disrupted localization manifests as poor contractile function will be more elusive. Spatial difficulties in the delivery of reducing equivalents from the sarcomere to the mitochondria, ${ }^{29}$ the (enigmatic) alterations in glycolytic flux, and mechanical impairment of actin-myosin interaction ${ }^{29}$ are all potential mechanisms.

The precise timing and profile of FHL2 decrease in human aortic stenosis remains to be elucidated. Donker and coworkers ${ }^{30}$ showed a transient decrease in FHL2 expression in dogs almost immediately after ventricular overload induced by atrioventricular block. They suggest that FHL2 reduction at that stage contributes to hypertrophy through derepression of the mitogen-activated protein kinase/ERK pathway. Our study groups were similar in terms of transvalvular gradients and showed FHL2 expression to be preserved during compensated stenosis, suggesting that FHL2 expression might be transiently restored by normalization of wall stress.

In summary, FHL2 expression and localization are preserved in human LV hypertrophy but disrupted in failing cardiomyocytes. Sequestration of the enzymes PFK2, AK, and CK-M by FHL2 appears responsible for their altered subcellular localization in human heart failure, which is associated with reduced activities of PFK2 and AK. This might yet prove to have important consequences for myocardial energy metabolism in heart failure.

We thank Dr R. H. Schule of the University of Freiberg, Germany, for provision of $\alpha$-FHL2 antibody.

\section{References}

1. Tarantini G, Buja P, Scognamiglio R, Razzolini R, Gerosa G, Isabella G, et al. Aortic valve replacement in severe aortic stenosis with left ventricular dysfunction: determinants of cardiac mortality and ventricular function recovery. Eur $J$ Cardiothorac Surg. 2003;24:879-85.

2. Arimura T, Hayashi T, Matsumoto Y, Shibata H, Hiroi S, Nakamura T, et al. Structural analysis of four and half LIM protein-2 in dilated cardiomyopathy. Biochem Biophys Res Commun. 2007;357:162-7.

3. Matsumoto Y, Hayashi T, Inagaki N, Takahashi M, Hiroi S, Nakamura T, et al. Functional analysis of titin/connectin N2-B mutations found in cardiomyopathy. J Muscle Res Cell Motil. 2005;26:367-74.

4. Lange S, Auerbach D, McLoughlin P, Perriard E, Schafer BW, Perriard JC, et al. Subcellular targeting of metabolic enzymes to titin in heart muscle may be mediated by DRAL/FHL-2. J Cell Sci. 2002;115:4925-36.

5. Wallimann T, Wyss M, Brdiczka D, Nicolay K, Eppenberger HM. Intracellular compartmentation, structure and function of creatine kinase isoenzymes in tissues with high and fluctuating energy demands: the "phosphocreatine circuit" for cellular energy homeostasis. Biochem J. 1992;281:21-40.

6. Dzeja PP, Redfield MM, Burnett JC, Terzic A. Failing energetics in failing hearts. Curr Cardiol Rep. 2000;2:212-7.

7. O'Brien PJ, Gwathmey JK. Myocardial Ca(2+)- and ATP-cycling imbalances in endstage dilated and ischemic cardiomyopathies. Cardiovasc Res. 1995;30:394-404.

8. Saupe KW, Spindler M, Tian R, Ingwall JS. Impaired cardiac energetics in mice lacking muscle-specific isoenzymes of creatine kinase. Circ Res. 1998;82: 898-907.

9. Depre C, Rider MH, Hue L. Mechanisms of control of heart glycolysis. Eur J Biochem. 1998;258:277-90.

10. Muller JM, Isele U, Metzger E, Rempel A, Moser M, Pscherer A, et al. FHL2, a novel tissue-specific coactivator of the androgen receptor. EMBO J. 2000;19:359-69.

11. Van Schaftingen E, Lederer B, Bartrons R. Hers HG A kinetic study of pyrophosphate: fructose-6-phosphate phosphotransferase from potato tubers. Application to a microassay of fructose 2,6-bisphosphate. Eur J Biochem. 1982;129:191-5.

12. Bishop C, Chu TM, Shihabi ZK. Single stable reagent for creatine kinase assay. Clin Chem. 1971;17:548-50.

13. Chu PH, Ruiz-Lozano P, Zhou Q, Cai C, Chen J. Expression patterns of FHL/ SLIM family members suggest important functional roles in skeletal muscle and cardiovascular system. Mech Dev. 2000;95:259-65.

14. Arber S, Hunter JJ, Ross JJ, Hongo M, Sansig G, Borg J, et al. MLP-deficient mice exhibit a disruption of cardiac cytoarchitectural organization, dilated cardiomyopathy, and heart failure. Cell. 1997;88:393-403.

15. Pashmforoush M, Pomies P, Peterson KL, Kubalak S, Ross JJ, Hefti A, et al. Adult mice deficient in actinin-associated LIM-domain protein reveal a developmental pathway for right ventricular cardiomyopathy. Nat Med. 2001;7:591-7.

16. Radke MH, Peng J, Wu Y, McNabb M, Nelson OL, Granzier H, et al. Targeted deletion of titin N2B region leads to diastolic dysfunction and cardiac atrophy. Proc Natl Acad Sci U S A. 2007;104:3444-9.

17. Kong Y, Shelton JM, Rothermel B, Li X, Richardson JA, Bassel-Duby R, et al. Cardiac-specific LIM protein FHL2 modifies the hypertrophic response to betaadrenergic stimulation. Circulation. 2001;103:2731-8.

18. Purcell NH, Darwis D, Bueno OF, Muller JM, Schule R, Molkentin JD. Extracellular signal-regulated kinase 2 interacts with and is negatively regulated by the LIM-only protein FHL2 in cardiomyocytes. Mol Cell Biol. 2004;24:1081-95.

19. McLoughlin P, Ehler E, Carlile G, Licht JD, Schafer BW. The LIM-only protein DRAL/FHL2 interacts with and is a corepressor for the promyelocytic leukemia zinc finger protein. J Biol Chem. 2002;277:37045-53.

20. Wojtas K, Slepecky N, von Kalm L, Sullivan D. Flight muscle function in Drosophila requires colocalization of glycolytic enzymes. Mol Biol Cell. 1997;8: 1665-75.

21. Saks VA, Tiivel T, Kay L, Novel-Chate V, Daneshrad Z, Rossi A, et al. On the regulation of cellular energetics in health and disease. Mol Cell Biochem. 1996; 160-161:195-208

22. Wixler V, Geerts D, Laplantine E, Westhoff D, Smyth N, Aumailley M, et al. The LIM-only protein DRAL/FHL2 binds to the cytoplasmic domain of several alpha and beta integrin chains and is recruited to adhesion complexes. J Biol Chem. 2000;275:33669-78.

23. Kupershmidt S, Yang IC, Sutherland M, Wells KS, Yang T, Yang P, et al. Cardiac-enriched LIM domain protein fhl 2 is required to generate $\mathrm{I}(\mathrm{Ks})$ in a heterologous system. Cardiovasc Res. 2002;56:93-103.

24. Nagueh SF, Shah G, Wu Y, Torre-Amione G, King NM, Lahmers S, et al. Altered titin expression, myocardial stiffness, and left ventricular function in patients with dilated cardiomyopathy. Circulation. 2004;110:155-62. 
25. Makarenko I, Opitz CA, Leake MC, Neagoe C, Kulke M, Gwathmey JK, et al. Passive stiffness changes caused by upregulation of compliant titin isoforms in human dilated cardiomyopathy hearts. Circ Res. 2004;95:708-16.

26. Filipeanu CM, Zhou F, Claycomb WC, Wu G. Regulation of the cell surface expression and function of angiotensin II type 1 receptor by Rab1-mediated endoplasmic reticulum-to-Golgi transport in cardiac myocytes. J Biol Chem. 2004;279:41077-84.

27. Vertessy BG, Orosz F, Kovacs J, Ovadi J. Alternative binding of two sequential glycolytic enzymes to microtubules. Molecular studies in the phosphofructokinase/aldolase/microtubule system. J Biol Chem. 1997;272:25542-6.
28. Parra J, Pette D. Effects of low-frequency stimulation on soluble and structurebound activities of hexokinase and phosphofructokinase in rat fast-twitch muscle. Biochim Biophys Acta. 1995;1251:154-60.

29. Srere PA, Knull HR. Location-location-location. Trends Biochem Sci. 1998;23 319-20.

30. Donker DW, Maessen JG, Verheyen F, Ramaekers FC, Spatjens RL, Kuijpers H, et al. Impact of acute and enduring volume overload on mechanotransduction and cytoskeletal integrity of canine left ventricular myocardium. Am J Physiol Heart Circ Physiol. 2007;292:H2324-32. 